\title{
Revisión
}

\section{Patogenia y expresión endoscópica de la infección por H. pylori en niños}

Publicado en Internet: 16-abril-2012

Iván Carabaño Aguado: carabano1975@hotmail.com
aServicio de Pediatría. Hospital Rey Juan Carlos. Móstoles, Madrid. España • bServicio de Pediatría. Hospital Infanta Elena. Valdemoro, Madrid. España • `Departamento de Anatomía Patológica. Fundación Jiménez Díaz. Madrid. España • dServicio de Pediatría. Hospital de Fuenlabrada.

Fuenlabrada, Madrid. España.
I. Carabaño Aguado ${ }^{a}$ E. La Orden Izquierdo ${ }^{b}$, C. Santonja Garriga ${ }^{c}$, F. J. Pelayo García ${ }^{b}$, L. Llorente Otones ${ }^{d}$, F. Manzarbeitia Arambarric

La infección por Helicobacter pylori es una de las causas más frecuentes de infección bacteriana crónica en el ser humano. A través de distintos factores patogénicos (adhesinas, ureasa, proteínas citotóxicas cagA y vacA) puede producir cambios que se pueden apreciar en una endoscopia digestiva alta. Estos incluyen la aparición de lesiones ulcerosas, mucosa en empedrado y palidez alternando con eritema, entre otros. Sus dianas preferentes en la edad pediátrica son el antro gástrico y el duodeno proximal. Este artículo hace una revisión de los hallazgos endoscópicos relacionados con la infección por $\mathrm{H}$. pylori en niños, así como de sus posibles causas y consecuencias clínicas.

\section{Pathogenesis and endoscopic findings in $H$. pylori infection in children}

- H. pylori

- Endoscopia

- Úlcera gástrica

- Úlcera duodenal
Key words:

- Endoscopy

- Gastric ulcus

- Duodenal ulcus
H. pylori infection is one of the most frequent chronic bacterial infections in human beings. Through different pathogenic factors (adhesins, urease, cagA and vacA cytotoxic proteins) it may produce mucosal changes that can be seen in an upper gastrointestinal endoscopy. These changes include, among others, ulcerative lesions, cobblestone mucosa and pallor alternating with erythema. Its favorite targets in children are the gastric antrum and proximal duodenum. This article reviews the endoscopic findings related to $\mathrm{H}$. pylori infection in children and their possible causes and clinical consequences.

\section{INTRODUCCIÓN}

Mucho se ha hablado, se habla y se hablará sobre la infección por Helicobacter pylori. Esta bacteria gram negativa microaerofílica puede presumir de contar con una revista científica íntegramente dedicada a ella. Su amplia extensión es determinante. De hecho, es una de las causas más frecuentes de infección bacteriana crónica en el ser humano ${ }^{1}$. En España, siguiendo un modelo de distribución propio de países desarrollados, se estima que la prevalencia en niños en edad escolar está en torno al 20-25\% $\%^{2}$. La edad media de adquisición de la infección, si extrapolamos los datos fiables de un estudio reciente en la población portuguesa, está en torno a los seis años y medio ${ }^{3}$.

Si bien la relación entre el $H$. pylori y la enfermedad gastroduodenal está bien establecida, no todos los individuos infectados desarrollan enfermedad ulcerosa, y menos aún cáncer gástrico, hecho que invita a pensar en la existencia de un factor individual de respuesta como condicionante de la gravedad clínica 4 .

El presente artículo supone una puesta al día ilustrada sobre los hallazgos endoscópicos derivados de la mencionada infección, siempre dentro del marco de la edad pediátrica. 


\section{DE LA INFECCIÓN A LAS ALTERACIONES ENDOSCÓPICAS}

En primer lugar, H. pylori, adquirido por una vía de contagio no definida completamente (fuentes posibles: oral-oral, gástrica-oral, fecal-oral), se fija a la mucosa del estómago y/o el duodeno a través de diversos tipos de adhesinas (BabA). Esta unión dispara el inicio del proceso inflamatorio. Una vez en la mucosa, mediante la producción de proteasas, arginasas y lipasas, comienza la ruptura de la integridad de la barrera mucosa, hecho potenciado por dos proteínas codificadas por el núcelo de 27 genes que confieren la agresividad de la bacteria. Estas son la toxina vacuolizante (vacA) y la proteína citotóxica responsable de la vacuolización de las células del epitelio gástrico (cagA), responsable además de afectación indirecta por estrés oxidativo. La prevalencia de estas dos toxinas aumenta con la edad, hecho que explica que la enfermedad ulcerosa sea menor en la edad pediátrica y mayor en la adulta. La seroprevalencia de ambas difiere en función de las poblaciones 5 .

H. pylori tiene capacidad para neutralizar la secreción ácida del estómago, a través de una gran producción de ureasa, con lo cual es capaz de elevar el pH local hasta cifras cercanas a 6,5, hecho que le facilita la síntesis proteica necesaria para su posterior división celular².

Otro factor patogénico cuyo alcance todavía está por ver es la hipermetilación aberrante del promotor $\mathrm{CpG}$ de las células gástricas. Esta, que ocurre durante las primeras fases de la infección, podría tener implicación en la aparición de cambios preneoplásicos, si bien su importancia es incierta ${ }^{6}$.

Queda por determinar por qué en niños la lesión suele ubicarse en el antro gástrico, y por qué suele condicionar nodularidad como cambio básico. No obstante, conviene tener en cuenta que el antro gástrico juega un papel primordial en la regulación de la secreción ácida. Recordemos que las células G, productoras de gastrina, están localizadas a lo largo de la mucosa gástrica y el duodeno. Estas células estimulan en último término a las células parietales, para que produzcan ácido clorhídrico.
En contraposición, las células $D$, que se ubican en la vecindad de las células $G$ antrales, se encargan de producir somatostatina, hormona que ejerce un efecto inhibidor sobre la secreción ácida. Desde un plano teórico, podríamos pensar que la destrucción de células $D$ en niños infectados por H. pylori dispararía la secreción ácida, si bien este hecho no está demostrado. Ni siquiera está claro que los niños infectados generen secreción ácida de forma distinta a los no infectados ${ }^{1}$. Podría existir un factor constitucional (por ejemplo, polimorfismos en el gen II-1) que explicase el ciclo de interacciones infección-secreción ácida-desarrollo de enfermedad. Estudios recientes apuntan que, al menos en la población china, los polimorfismos II-1B-511TT/31CC se asocian a afectación mucosa gástrica importante ${ }^{7}$. En todo caso, la afectación antral se asocia con menor riesgo de cáncer que la corpofúndica². La respuesta a la infección en niños suele evidenciar en el plano microscópico una menor respuesta neutrofílica que la objetivada en adultos. Esta respuesta atemperada puede explicar la menor asociación con ulcus y gastritis atrófica en la edad pediátrica ${ }^{4}$.

\section{HALLAZGOS ENDOSCÓPICOS MÁS FRECUENTES}

La endoscopia digestiva alta es la técnica de elección a la hora de diagnosticar la enfermedad ulcerosa, incluso en niños pequeños. Sus indicaciones vienen delimitadas por los criterios de organicidad propuestos hace diez años por Chelimsky y Czinn ${ }^{8}$ (Tabla 1), y que sirvieron como punto de partida para los criterios Roma III $^{9}$. Estos criterios han mostrado ser una herramienta efectiva a la hora de discriminar pacientes con organicidad manifiesta ${ }^{10}$. A través de la endoscopia digestiva alta se podrá obtener biopsias para examen histológico y para hacer el test de la ureasa ${ }^{11}$. Ambas tienen una especificidad similar, pero el examen histológico es más sensible a la hora de diagnosticar la infección ${ }^{4,11}$. H. pylori se puede evidenciar a través de dos tinciones: Warthin-Starry (Fig. 1) y Giemsa (Fig. 2). El material histológico se puede utilizar para hacer cultivo y estudio de resistencias. 


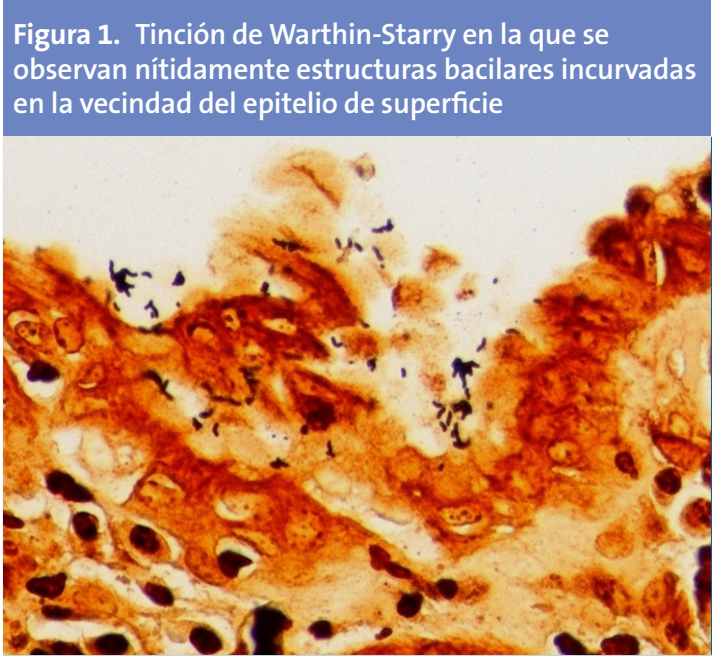

La infección por H. pylori puede dar lugar al espectro de lesiones que se exponen en la Tabla 2,2,12.

La diana de afectación principal en las infecciones por H. pylori se centra en el antro gástrico ${ }^{1}$. Se ha descrito ampliamente la asociación entre la infección por H. pylori y la aparición de múltiples nódulos en el antro, de 2-5 mm de diámetro cada uno, hecho que confiere un aspecto "en empedrado" (Fig. 3). A este se ha aludido también como "gastritis nodular", "gastritis micronodular" o "hiperplasia linforreticular"1. Se desconoce si la gastritis nodular tiene correlato clínico o si, por el contrario, es un hallazgo intrascendente ${ }^{2}$. Aun así, cabe destacar que es una lesión muy característica, y que se da con mayor frecuencia en niños que en adultos ${ }^{4}$. La nodularidad antral tiene alta especificidad (94-95\%) y valor predictivo

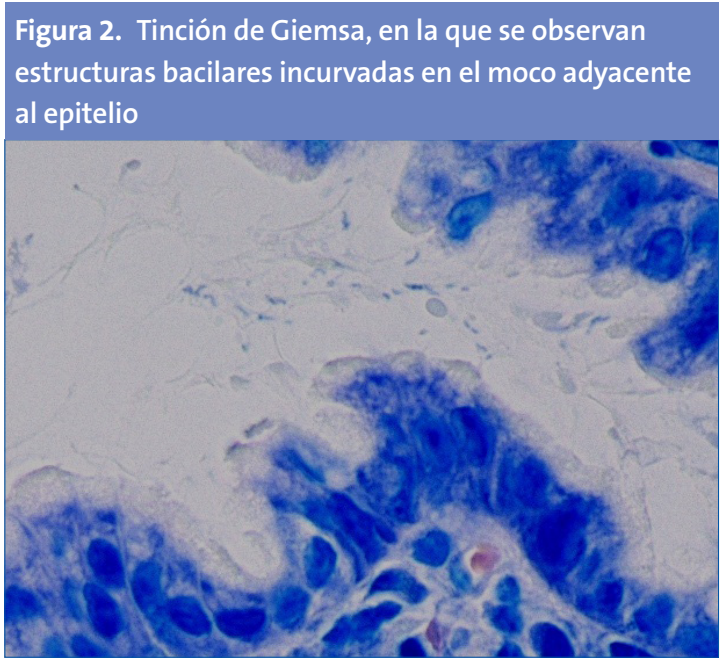

positivo (97-99\%) con respecto a la infección por H. pylori en la edad pediátrica, y puede asociarse o no a la aparición de úlceras duodenales². Desde el punto de vista histológico, la nodularidad sugiere una combinación de aumento en los folículos linfoides e infiltrado por polimorfonucleares $^{13,14}$ (Fig. 4). Se desconoce la causa exacta que dispara la transformación nodular mucosa, y si esta rige una periodicidad estacional o temporal. ${ }^{1}$.

La apariencia endoscópica de las úlceras pépticas localizadas en el estómago depende de su estadio de evolución. Las úlceras activas se muestran redondeadas $u$ ovales, con una base blanquecina compuesta por fibrina y detritus, y bordes eritematosos o sobreelevados ${ }^{1,2,4}$ (Fig. 5). Ante la visualización de cualquier úlcera, se ha de constatar: tama-

Tabla 1. Criterios diagnósticos de dispepsia orgánica

\begin{tabular}{|l|l|}
\hline \multicolumn{1}{|c|}{ Mayores } & \multicolumn{1}{|c|}{ Menores } \\
\hline Epigastralgia & Anorexia y pérdida de peso \\
& Despertares nocturnos \\
& Acidez \\
& Regurgitación oral \\
& Náusea crónica \\
& Eructos o hipo exagerados \\
& Saciedad precoz \\
& Dolor periumbilical \\
& Contexto familiar de enfermedad ulceropéptica, dispepsia o colon \\
& irritable \\
\hline $\begin{array}{l}\text { La presencia de dos criterios mayores, o de uno mayor y dos menores, o de cuatro menores, son indicaciones para realizar una } \\
\text { endoscopia digestiva alta. }\end{array}$
\end{tabular}


Figura 3. Nodularidad antral florida

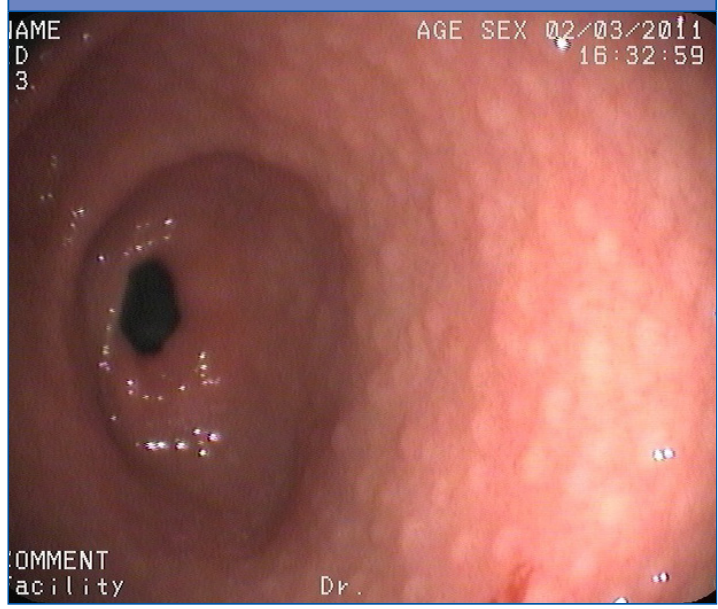

ño, localización, número, lesiones asociadas y signos de actividad hemorrágica. En cuanto a su tamaño, se ha descrito que el 90\% mide al menos $3 \mathrm{~cm}^{2}$. Las úlceras gástricas suelen localizarse alrededor de la banda transversal, y centradas por la cisura angularis. A dicho nivel, las fibras musculares se disponen a modo de falla geológica. La mucosa perilesional suele disponer los pliegues en forma radial sobre el ulcus².

Aun así, la apariencia endoscópica es a veces engañosa, pues imagen normal y ausencia de gastritis no son términos sinónimos. De ahí la importancia de extraer un mapeo de biopsias para procesamiento histológico cuando exista una firme sospe-

Figura 5. Úlcera antral de nicho inactivo cubierta por fibrina, en la vecindad de la cisura angularis

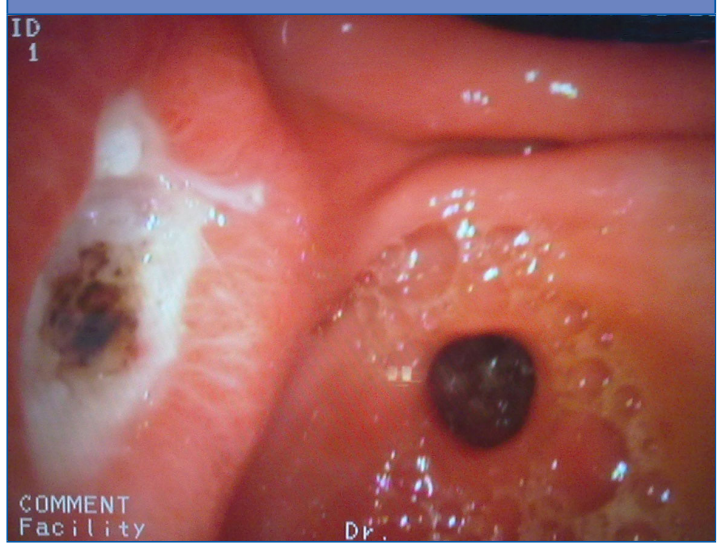

Figura 4. Contexto histopatológico de la Fig. 3. Mucosa antral con infiltrado inflamatorio mixto netrofílicolinfoplasmocitario. Tinción con hematoxilina-eosina

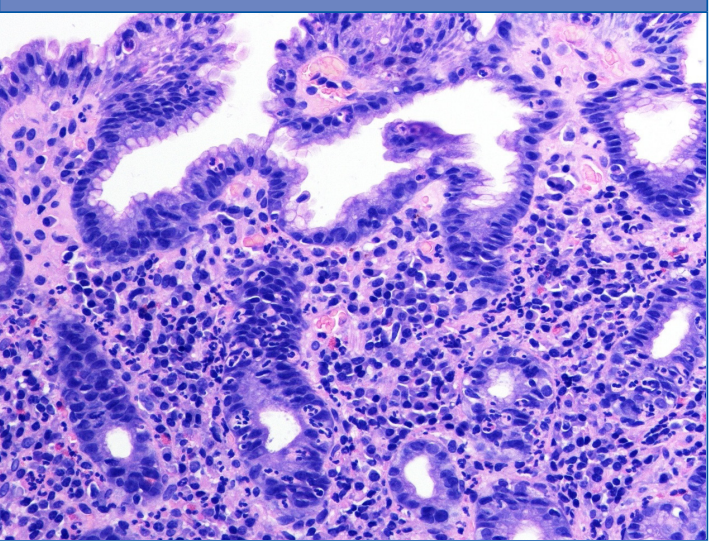

cha clínica. Las mismas servirán para facilitar un cultivo con estudio de resistencias ${ }^{13}$.

En otras ocasiones, a nivel gástrico se objetiva un tenue parcheado eritematoso alternando con zonas de palidez. Cambios similares se pueden apreciar en el duodeno (Fig. 6), donde también se puede apreciar nodularidad y nichos de úlcera ${ }^{13,14}$ Aunque a veces son lineales o irregulares, las úlceras duodenales suelen tener contornos nítidos y forma regular; su ubicación más frecuente es el bulbo, y su tamaño suele ser menor de $1 \mathrm{~cm}^{2}$.

Surge la controversia de si es o no necesario tratar la enfermedad no ulcerosa. En principio, la gastritis por $H$. pylori no es una indicación clara de trata-

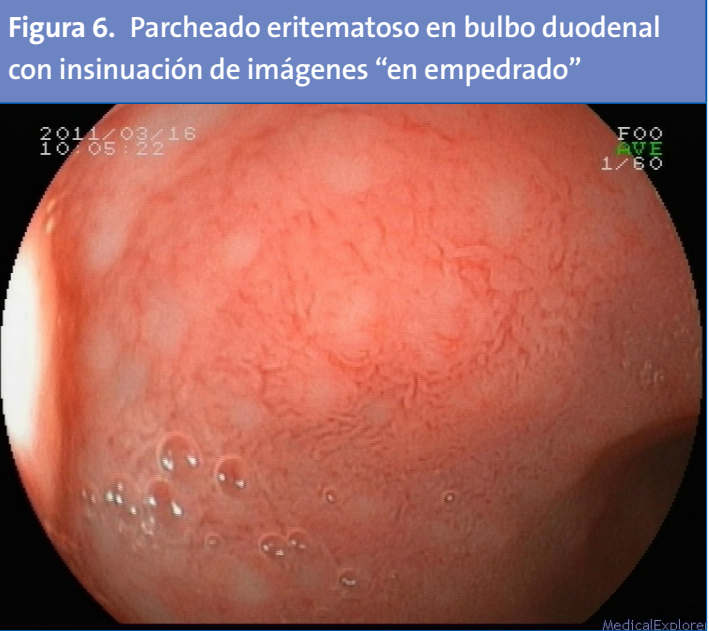


Tabla 2. Hallazgos endoscópicos en la infección por H. pylori

\begin{tabular}{|l|l|}
\hline \multicolumn{1}{|c|}{ Estómago } & \multicolumn{1}{c|}{ Duodeno } \\
\hline Mucosa normal & Úlcera duodenal \\
Engrosamiento de pliegues & Parcheado eritematoso con adelgazamiento duodenal \\
Parcheado eritematoso con adelgazamiento gástrico & Nodularidad duodenal \\
Nodularidad antral & Linfoma MALT \\
Úlcera gástrica & \\
Linfoma MALT & \\
\hline
\end{tabular}

miento, pero sí lo es la enfermedad ulcerosa ${ }^{15,16}$. En la guía de la NASPGHAN/ESPGHAN se dice que, en ausencia de ulcus, si se demuestra mediante biopsia la infección por H. pylori, debe considerarse el tratamiento. Además, concluyen que la decisión de tratar las gastritis está sujeta a juicio del clínico junto con el paciente y la familia, teniendo en cuenta los riesgos potenciales y los beneficios, de forma individua, con todo el componente subjetivo que esto conlleva ${ }^{15}$. El grupo de Buonovolontá ${ }^{16}$ establece dos grupos de niños con gastritis: uno sometido a tratamiento erradicador y otro no so-

\section{BIBLIOGRAFÍA}

1. Mourad-Baars P, Hussey S, Jones NL. Helicobacter pylori infection in childhood. Helicobacter. 2010; 15:53-9.

2. Martínez Gómez MJ, Camarero Salces C. Gastritis y enfermedad ulceropéptica. En: Argüelles Martín F, García Novo MD, Pavón Belinchón P, Román Riechmann E, Silva García G, Sojo Aguirre A. Tratado de gastroenterología, hepatología y nutrición de la SEGHNP. Madrid: Ergón; 2011. p. 190-7.

3. Oleastro M, Pelerito A, Nogueira P, Benoliel J, Santos A, Cabral J, et al. Prevalence and incidence of Helicobacter pylori infection in a healthy pediatric population in the Lisbon area. Helicobacter. 2011;16:363-72.

4. Pacifico L, Anania C, Osborn JF, Ferraro F, Chiesa C. Consequences of Helicobacter pylori in children. World J Gastroenterol. 2010;16:5181-94.

5. Wnuk M, Myszka A, Lewinska A, Tokarz I, Solarska K. Helicobacter pylori cagA gene polymorphism affects the total antioxidant capacity of human saliva. Helicobacter. 2010;15:53-7.

6. Shin SH, Park SY, Ko JS, Kim N, Kang GH. Aberrant CpG island hypermethylation in pediatric gastric mucosa metido a tratamiento. Y comprueba que los síntomas no desaparecen a lo largo del año siguiente en los niños en los que se ha erradicado el germen, a pesar de que se haya curado la gastritis. ¿Qué hacer, por tanto? La polémica sigue abierta a futuras investigaciones.

\section{CONFLICTO DE INTERESES}

Los autores declaran no presentar conflictos de intereses en relación con la preparación y publicación de este artículo.

in association with Helicobacter pylori infection. Arch Pathol Lab Med. 2011;135:759-65.

7. Li J, Wang F, Zhou O, Ou Z, Jia H, Deng X, et al. II-1 polymorphisms in children with peptic synptoms in South China. Helicobacter. 2011;16:246-51.

8. Chelimsky G, Czinn SJ. Techniques for the evaluation of dispepsia in children. J Clin Gastroenterol. 2001; 33:11-3.

9. Rasquin A, Di Lorenzo C, Forbes D, Guiraldes E, Hyams JS, Staiano A, et al. Childhood functional disorders: child/adolescent. Gastroenterol. 2006;130:1527-37.

10. Tam YH, Chan KW, To KF, Cheung ST, Mou JW, Pang $\mathrm{KK}$, et al. Impact of pediatric Rome III criteria of functional dyspepsia on the diagnostic yield of upper endoscopy and predictors for a positive endoscopic finding. J Pediatric Gastroenterol Nutr. 2011;52:387-91.

11. Roma-Giannikou E, Roubani A, Sgouras DN, Panayiotou J, van-Vliet C, Polyzos A et al. Endoscopic tests for the diagnosis of Helicobacter pylori infection in children: validation of rapid ureasa test. Helicobacter. 2010;15:227-32.

12. Hoepler W, Hammer K, Hammer J. Gastric phenotype in children with Helicobacter pylori infection under- 
going upper endoscopy. Scand J Gastroenterol. 2011;46:293-8.

13. Yakoob MY, Hussainy AS. Chronic gastritis and Helicobacter pylori: a histopathological study of gastric mucosal biopsies. J Coll Physicians Surg Pak. 2010;20:773-5.

14. Gonul CD, Bilge C, Gazi KA, Filiz K. Duodenal nodularity in children: a clinical and pathologic study of 17 cases. Indian J Pathol Microbiol. 2011;54:312-7.
15. Koletzko S, Jones NL, Goodman KJ, Gold B, Rowland $M$, Cadranel S, et al. Evidence-based guidelines from ESPGHAN and NASPGHAN for Helicobacter pylori infection in children. JPGN. 2011;53:230-43.

16. Buonavolontà R, Miele E, Russo D, Vecchione R, Straiano A. Helicobacter pylori chronic gastritis in children: to erradicate or not to erradicate? J Pediatr. 2011;159:50-6. 